\title{
A combination of platelet features allows detection of early-stage cancer
}

Citation for published version (APA):

Sabrkhany, S., Kuijpers, M. J. E., van Kuijk, S. M. J., Sanders, L., Pineda, S., Damink, S. W. M. O., Dingemans, A-M. C., Griffioen, A. W., \& Egbrink, M. G. A. O. (2017). A combination of platelet features allows detection of early-stage cancer. European Journal of Cancer, 80, 5-13.

https://doi.org/10.1016/j.ejca.2017.04.010

Document status and date:

Published: 01/07/2017

DOI:

10.1016/j.ejca.2017.04.010

Document Version:

Publisher's PDF, also known as Version of record

Document license:

Taverne

Please check the document version of this publication:

- A submitted manuscript is the version of the article upon submission and before peer-review. There can be important differences between the submitted version and the official published version of record.

People interested in the research are advised to contact the author for the final version of the publication, or visit the DOI to the publisher's website.

- The final author version and the galley proof are versions of the publication after peer review.

- The final published version features the final layout of the paper including the volume, issue and page numbers.

Link to publication

\footnotetext{
General rights rights.

- You may freely distribute the URL identifying the publication in the public portal. please follow below link for the End User Agreement:

www.umlib.nl/taverne-license

Take down policy

If you believe that this document breaches copyright please contact us at:

repository@maastrichtuniversity.nl

providing details and we will investigate your claim.
}

Copyright and moral rights for the publications made accessible in the public portal are retained by the authors and/or other copyright owners and it is a condition of accessing publications that users recognise and abide by the legal requirements associated with these

- Users may download and print one copy of any publication from the public portal for the purpose of private study or research.

- You may not further distribute the material or use it for any profit-making activity or commercial gain

If the publication is distributed under the terms of Article $25 \mathrm{fa}$ of the Dutch Copyright Act, indicated by the "Taverne" license above, 


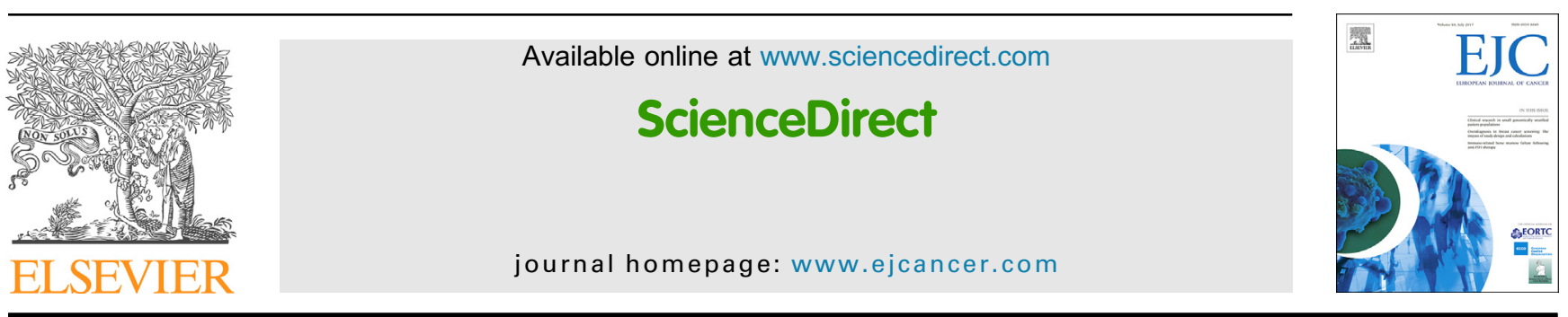

Original Research

\title{
A combination of platelet features allows detection of early-stage cancer
}

\author{
Siamack Sabrkhany ${ }^{\mathrm{a}}$, Marijke J.E. Kuijpers ${ }^{\mathrm{b}}$, Sander M.J. van Kuijk ${ }^{\mathrm{c}}$, \\ Linda Sanders ${ }^{a}$, Sharo Pineda ${ }^{a}$, Steven W.M. Olde Damink ${ }^{\mathrm{d}}$, \\ Anne-Marie C. Dingemans ${ }^{e}$, Arjan W. Griffioen ${ }^{\text {f }}$, \\ Mirjam G.A. oude Egbrink ${ }^{a, *}$
}

\footnotetext{
${ }^{a}$ Department of Physiology, Cardiovascular Research Institute Maastricht, Maastricht University Medical Center+, Maastricht, The Netherlands

${ }^{\mathrm{b}}$ Department of Biochemistry, Maastricht University Medical Center+, Maastricht, The Netherlands

${ }^{\mathrm{c}}$ Department of Clinical Epidemiology and Medical Technology Assessment, Maastricht University Medical Center+. Maastricht, The Netherlands

${ }^{\mathrm{d}}$ Department of Surgery, Maastricht University Medical Center+, Maastricht, The Netherlands

${ }^{\mathrm{e}}$ Department of Pulmonology, Maastricht University Medical Center+, Maastricht, The Netherlands

${ }^{\mathrm{f}}$ Department of Medical Oncology, Angiogenesis Laboratory, VU Medical Center, Amsterdam, The Netherlands
}

Received 21 February 2017; received in revised form 2 April 2017; accepted 5 April 2017

Available online 17 May 2017

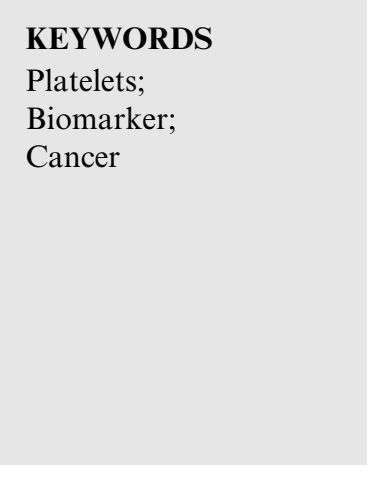

\begin{abstract}
Background: Detection of early-stage cancer significantly improves patient survival. As platelets play an important role in cancer progression, we aimed to investigate whether platelets can be used for the discovery of early-stage cancer.

Methods: Patients with lung $(n=86)$ or head of pancreas $(n=42)$ cancer were included, as were healthy sex- and age-matched controls $(\mathrm{n}=92)$. Blood was collected before initiation of treatment. Platelet count, volume and activation status were quantified in whole blood. Next, concentrations of vascular endothelial growth factor, platelet-derived growth factor, platelet factor 4, thrombospondin-1 and connective tissue-activating peptide III were measured in both platelets and plasma. Using the results, two multivariable diagnostic models were developed and internally validated.

Findings: Multiple platelet features, including platelet count, volume and protein content, were significantly changed in lung and head of pancreas cancer patients. However, the pattern
\end{abstract}

\footnotetext{
* Corresponding author: Department of Physiology, Maastricht University, P.O. Box 616, 6200 MD Maastricht, The Netherlands. Fax: +31 43 3884166.

E-mail address: m.oudeegbrink@maastrichtuniversity.nl (M.G.A. oude Egbrink).
} 
of changes differed between both groups. The diagnostic model developed for lung cancer discriminated very well between patients and controls (AUC $=88.7 \%$ ). Addition of smoking as a variable significantly increased the AUC of the model to $94.5 \%$. The diagnostic model for head of pancreas cancer also performed well (AUC $=82.7 \%$ ). Both models were internally validated, resulting in optimism-corrected AUC's of $86.8 \%$ and $80.8 \%$, respectively.

Interpretation: In patients with lung or head of pancreas cancer, several platelet characteristics are changed compared to healthy sex- and age-matched controls. A cancer type-specific combination of these platelet features can be used to discriminate between patients with early-stage cancer and healthy individuals.

(C) 2017 Elsevier Ltd. All rights reserved.

\section{Introduction}

Detection of cancer in its early stages radically improves the effectiveness of available treatment and overall prognosis of patients [1]. Up to now, studies searching for biomarkers are mostly based on blood plasma or serum parameters. A limitation of this approach is that platelets and their content are neglected [2].

Circulating platelets contain numerous proteins, including growth factors, chemokines and proteases, which are synthesised by megakaryocytes or absorbed from the blood by the platelets themselves [3]. Therefore, the presence of a growth factor-producing tumour can influence platelet content. Concentrations of angiogenic factors, like vascular endothelial growth factor (VEGF) and platelet-derived growth factor (PDGF), and the angiostatic platelet factor 4 (PF4) have been reported to be higher in platelets of patients with cancer than in platelets of healthy individuals $[4,5]$. Another recent study suggests that platelet mRNA profiles are also altered in patients with cancer, enabling discrimination between cancer patients and healthy individuals [6]. In addition, platelets may become activated systemically or within the tumour, potentially resulting in release of platelet content into the circulation [3]. Next to platelet content and platelet activation, platelet count is also frequently changed in cancer patients [7]. Tumours can increase platelet production by secretion of thrombopoietic cytokines, leading to paraneoplastic thrombocytosis $[7,8]$.

Altogether, data from literature suggest that several platelet characteristics are affected in cancer patients. These features, either alone or in combination, may be useful tools in the detection of (early stages of) cancer. It was the aim of the present study to investigate, in two different groups of cancer patients, whether and how platelet features are changed in the presence of a tumour. In addition, we succeeded to combine these features into two internally validated diagnostic models, one for lung cancer and another one for head of pancreas cancer, to discriminate between patients and healthy individuals.

\section{Methods}

\subsection{Study design and participants}

This study was performed in accordance with the Declaration of Helsinki and approved by the medical ethical committee of Maastricht University Medical Center+. Informed consent was obtained from all participants. Patients with clinically established and histologically proven untreated primary lung $(\mathrm{n}=86)$ or head of pancreas cancer $(\mathrm{n}=42$, including pancreas head cancer $[n=28]$, distal cholangiocarcinoma $[n=8]$ and duodenumcarcinoma $[\mathrm{n}=6]$ ), that were eligible for surgical resection, were included between July 2012 and October 2014. Exclusion criteria were previous history of cancer, neo-adjuvant chemotherapy or radiotherapy, use of platelet-influencing drugs such as aspirin, blood or platelet transfusion during the previous 14 days, active inflammatory disease, non-healing ulcers or fractures. Staging was performed in accordance with the tumour-node-metastasis (TNM) classification (version 7) of the Union for International Cancer Control [9]. A sex- and age-matched healthy control population for both cancer groups was included as well (Table 1).

\subsection{Procedures}

Blood from all patients and healthy individuals was collected. In case of cancer patients, sampling occurred within 1 week before initiation of treatment. To prevent platelet activation during blood collection and sample preparation, blood was collected as described before [10].

The procedures for quantification of platelet count, volume and activation status, as well as concentrations of various growth factors and chemokines in platelets and plasma, are described extensively in the Appendix. In short, platelet count and volume were determined in whole blood with a Beckman coulter counter, and platelet activation was quantified in whole blood using flow cytometry. The remaining blood was centrifuged several times to obtain platelet-free plasma (PFP) and 
Table 1

Patient demographics and clinical profiles.

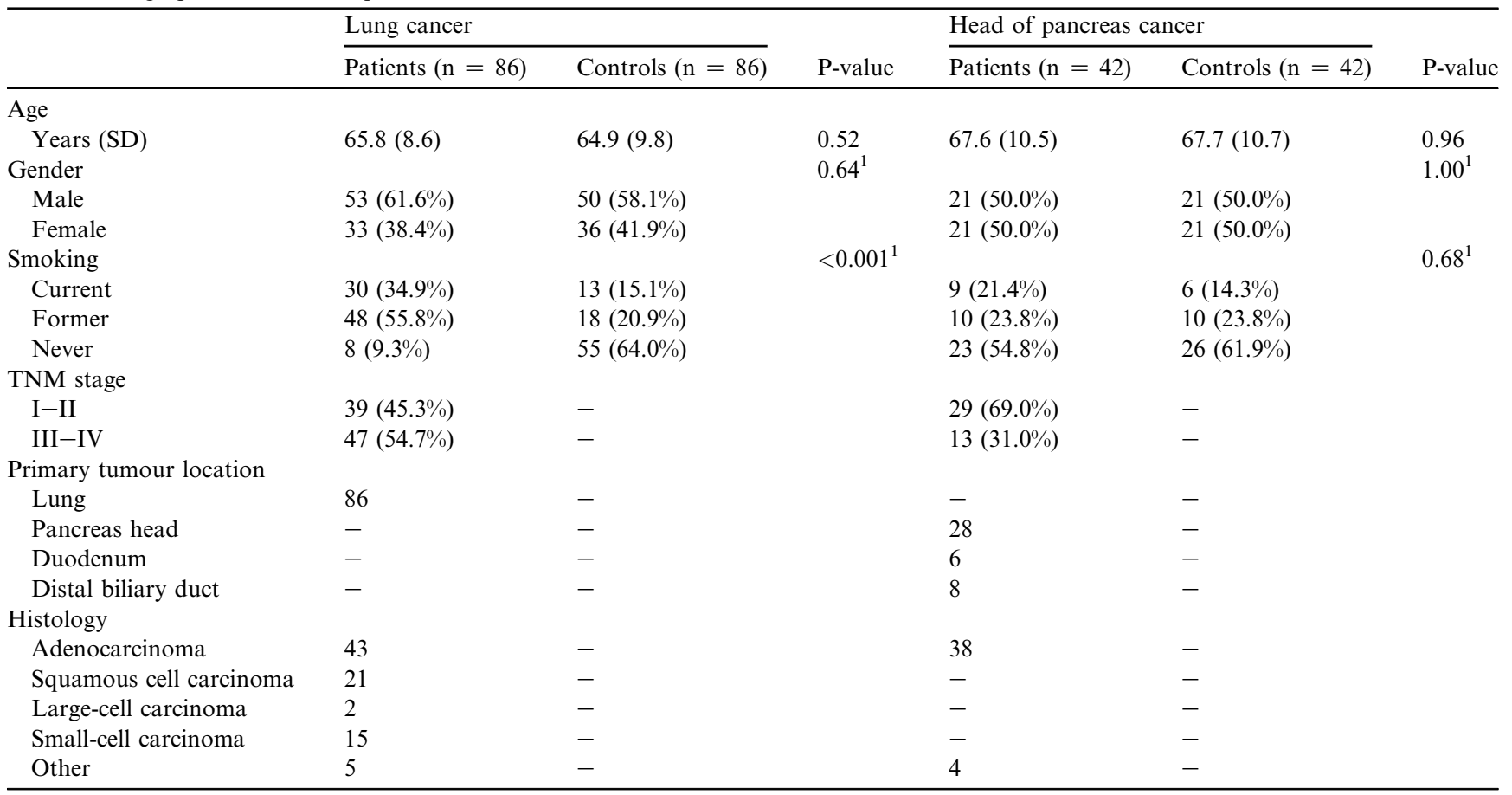

Patient baseline characteristics were compared to data from the healthy control groups using the t-test for continuous variables and ${ }^{1}$ Pearson's chisquare test for categorical variables. P-values less than 0.05 were considered statistically significant.

$\mathrm{SD}=$ standard deviation; TNM, tumour-node-metastasis.

platelet pellet. In both platelets and PFP, the concentrations of the angiogenic proteins VEGF and PDGF, as well as the angiostatic factors PF4, thrombospondin1 (TSP-1) and connective tissue-activating peptide III (CTAPIII) were measured using specific human DuoSet ELISA assays (R\&D Systems, Abingdon, United Kingdom). ELISA measurements in all patient and matching control samples were performed using the same assay, simultaneously and in the same institution.

\subsection{Statistics}

Statistical analyses were performed using SPSS (version 22; SPSS Inc, Chicago, USA) and $\mathrm{R}$ (version 3.2.2, R core development team). Data are presented as means with standard errors of the mean unless otherwise indicated. Patient data were compared to data from the healthy control groups using the t-test for continuous variables and Pearson's chi-square test for categorical variables. Spearman's rank correlation was used to test the association between variables. P-values less than 0.05 were considered statistically significant.

\subsection{Development of diagnostic models}

For both cancer groups, logistic regression was used to estimate diagnostic models. All platelet parameters were added, after which stepwise backward elimination was used to arrive at a more parsimonious model. The derivation of the diagnostic model for lung cancer was repeated to also include smoking status (current, former and never) to assess its performance when combined with arguably the strongest known predictor for lung cancer.

Model performance was assessed by Nagelkerke's $\mathrm{R}^{2}$ statistic and by quantifying discrimination. Nagelkerke's $\mathrm{R}^{2}$ can be used to quantify the predictive strength of a diagnostic model. Discrimination is the model's ability to distinguish between those that have cancer and those who do not. Discrimination was quantified by the area under the receiver operating characteristic curve (AUC). In addition, boxplots were constructed to visually assess how the diagnostic model distinguishes cases from controls.

Since the number of predictors that was entered in the models was higher than recommended using the 10 events-per-variable rule of thumb, the risk of overfitting the diagnostic models was relatively high. Therefore, the models were internally validated using standard bootstrapping techniques [11]. In this step, 1000 bootstrap samples of the original data were drawn, and similar diagnostic models were estimated using these data. The average difference in diagnostic model performance between the bootstrap sample and the original sample provided estimates of optimism in the performance measures. These measures of optimism were subsequently subtracted from the performance measures we computed, to reflect the likely performance of the models in future patients. 


\section{Results}

Blood was collected from 86 lung cancer patients, 42 patients diagnosed with head of pancreas cancer and a total of 92 healthy individuals. Importantly, the latter group contained individuals that were matched with respect to gender and age to both groups of cancer patients. All cancer patients were treatment naive at the moment of blood sampling. Detailed characteristics of the study populations are presented in Table 1 . The lung cancer group comprised significantly more (current and former) smokers than the control group. In the healthy individuals, both gender and age appeared to be related to some of the platelet characteristics. Mean platelet count appeared to be higher in females than males (235 versus $201 \times 10^{9} / \mathrm{L} ; \mathrm{p}<0.01$ ), while age showed to be negatively correlated with platelet concentrations of PDGF (Spearman's r: -0.43 ; p $<0.0001$ ), PF4 (r: $-0.31 ; \mathrm{p}<0.01)$ and CTAPIII (r: $-0.21 ; \mathrm{p}<0.05$ ).

\subsection{Platelet characteristics in patients with untreated lung cancer}

Platelet counts were not different as compared to controls in patients with early-stage (stage I-II) lung cancer, but significantly increased in case of advanced (stage III-IV) lung cancer (Fig. 1A). Mean platelet volume (MPV) was higher in early-stage (stage I-II) lung cancer only (Fig. 1B).

Concentrations of VEGF, PDGF, CTAPIII, PF4 and TSP-1 in platelets appeared to differ significantly between lung cancer patients and controls. VEGF concentration was significantly elevated in platelets of both stage I-II and stage III-IV patients (Fig. 1C), while platelet PDGF concentration was elevated in stage I-II only (Fig. 1D). In contrast, concentrations of CTAPIII, PF4 and TSP-1 were similar to control in early-stage cancer but significantly reduced in platelets of patients with advanced lung cancer (Fig. 1E-G).
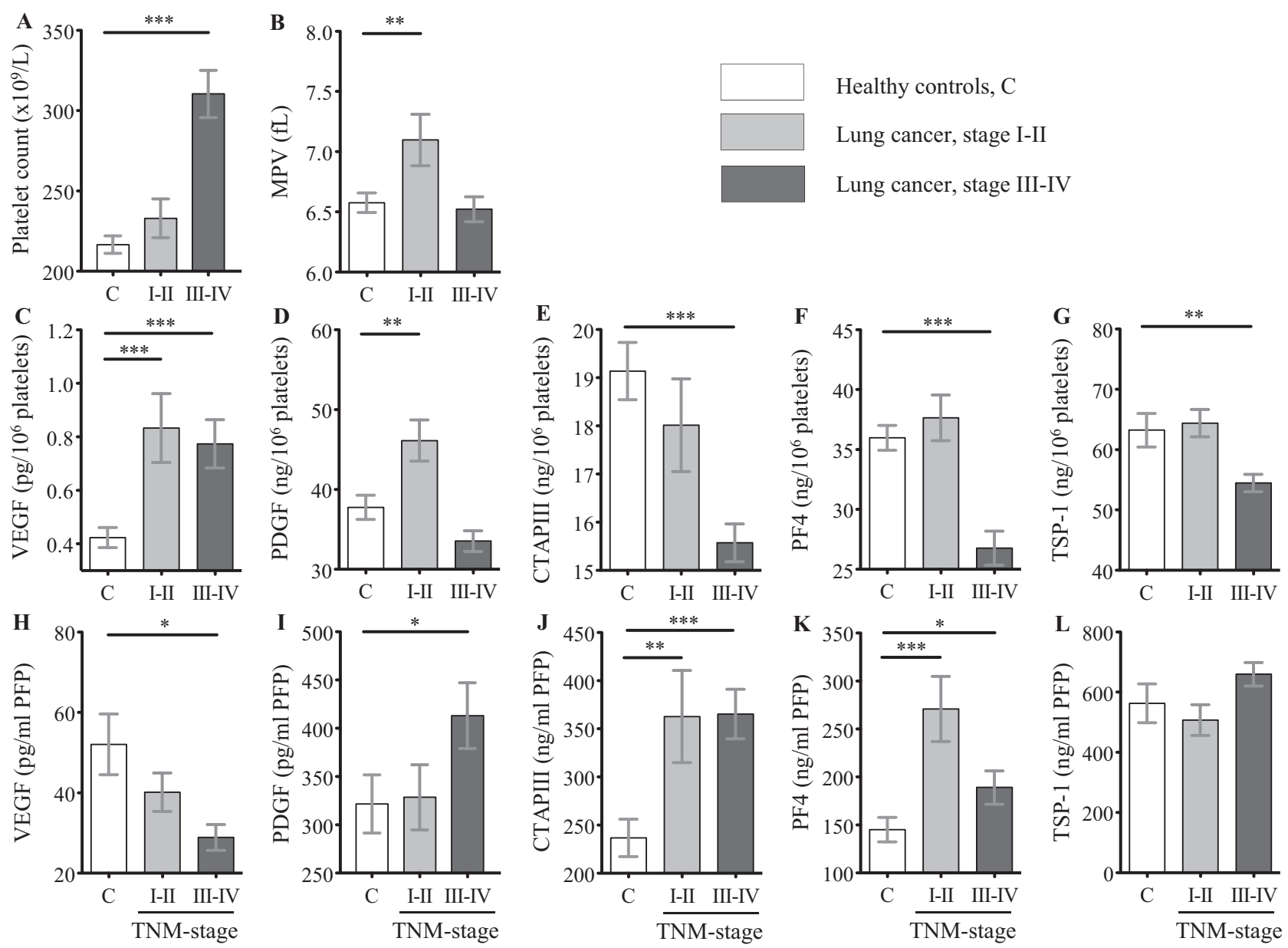

Fig. 1. Lung cancer is associated with changes in platelet count, mean platelet volume (MPV), growth factor content and activation. Platelet characteristics were measured in healthy controls $(\mathrm{C}, \mathrm{n}=86)$ and in patients with lung cancer, that were subdivided according to TNM-stage (I-II, n = 39; III-IV, n = 47). (A-B) Whole blood platelet count and MPV were measured after blood collection. (C-L) Platelets and platelet-free plasma (PFP) were isolated from whole blood. Concentrations of VEGF, PDGF, CTAPIII, PF4 and TSP-1 were determined in platelets $(\mathrm{C}-\mathrm{G})$ and PFP $(\mathrm{H}-\mathrm{L})$. Data are presented as means with standard errors of the means. ${ }^{*} \mathrm{p}<0.05$; ${ }^{* *} \mathrm{p}<0.01 ;{ }^{* * *} \mathrm{p}<0.001$. TNM, tumour-node-metastasis; PDGF, platelet-derived growth factor; PF4, platelet factor 4; CTAPIII, connective tissue-activating peptide III; TSP-1, thrombospondin-1; VEGF, vascular endothelial growth factor. 
Strikingly, changes in platelet content did not correspond with concentration changes of the same proteins in PFP (Fig. 1H-L). VEGF concentration was decreased and PDGF concentration increased in advanced stage lung cancer (Fig. $1 \mathrm{H}$ and I). CTAPIII and PF4 concentrations (Fig. $1 \mathbf{J}$ and $\mathrm{K}$ ) were elevated in plasma of both early- and advanced-stage lung cancer patients, while TSP-1 plasma concentration was not changed in any of the lung cancer patient groups (Fig. 1L). To exclude the possibility that the increased PDGF, CTAPIII and PF4 plasma levels in patients result from ex vivo platelet activation (by blood collection and preparation), we determined P-selectin expression and integrin $\alpha_{\mathrm{IIb}} \beta_{3}$ activation on unstimulated platelets of patients and controls by flow cytometry. No differences between patients and controls were found (Supplemental Fig. 1A and B). Furthermore, the platelets were similarly responsive to in vitro activation (Supplemental Fig. 1C and D). This suggests that the increase in PDGF, CTAPIII and PF4 plasma concentrations in patients with lung cancer is mainly due to systemic or intratumoural platelet activation, leading to secretion of their content.

\subsection{A combination of platelet features discriminates lung cancer patients from controls}

Data from 172 individuals (86 patients with lung cancer and 86 sex- and age-matched controls) were available for multivariable modelling. All measured parameters were entered into the model: platelet count, MPV and concentrations of VEGF, PDGF, PF4, CTAPIII and TSP-1 in platelets and PFP. The stepwise backward elimination procedure yielded a diagnostic model consisting of a final set of six parameters (platelet count, MPV, PF4 and CTAPIII concentrations in platelets, and CTAPIII and TSP-1 concentrations in PFP). Nagelkerke's $\mathrm{R}^{2}$ of the diagnostic model was 0.572 , indicating that the model fits the data well. Fig. 2A shows the performance of the model with an area under the curve (AUC) of $88.7 \%$ (95\% confidence interval [CI] 84.1-93.2). The optimismcorrected AUC (i.e. the likely value of the AUC when applied to future patients) was $86.8 \%$. This demonstrates that the model discriminated very well between lung cancer cases and controls in our study population. This was confirmed by the boxplots of predicted probability by the diagnostic model (Fig. 2B). The distribution of probabilities of lung cancer cases and controls were almost separated; there was only little overlap between controls on the one hand and stage I-II and III-IV lung cancer patients on the other. Addition of smoking as a variable significantly increased the predicted probability of the diagnostic model $(\mathrm{p}<0 \cdot 001, \mathrm{AUC}=94.5 \%[95 \%$ CI 91.0-98.1]; Fig. 2A).

\subsection{A platelet-based marker for the presence of head of pancreas cancer}

In patients diagnosed with head of pancreas cancer, several platelet parameters were found to be substantially different from the control group as well. Platelet count was significantly elevated in stage I-II (Fig. 3A) and MPV in stage III-IV cancer (Fig. 3B). VEGF concentration was significantly increased in platelets of patients with early- and late-stage head of pancreas cancer (Fig. 3C), while no differences in platelet PDGF, CTAPIII, PF4 and TSP-1 concentrations were observed (Supplemental Fig. 2A-D). In PFP, PDGF
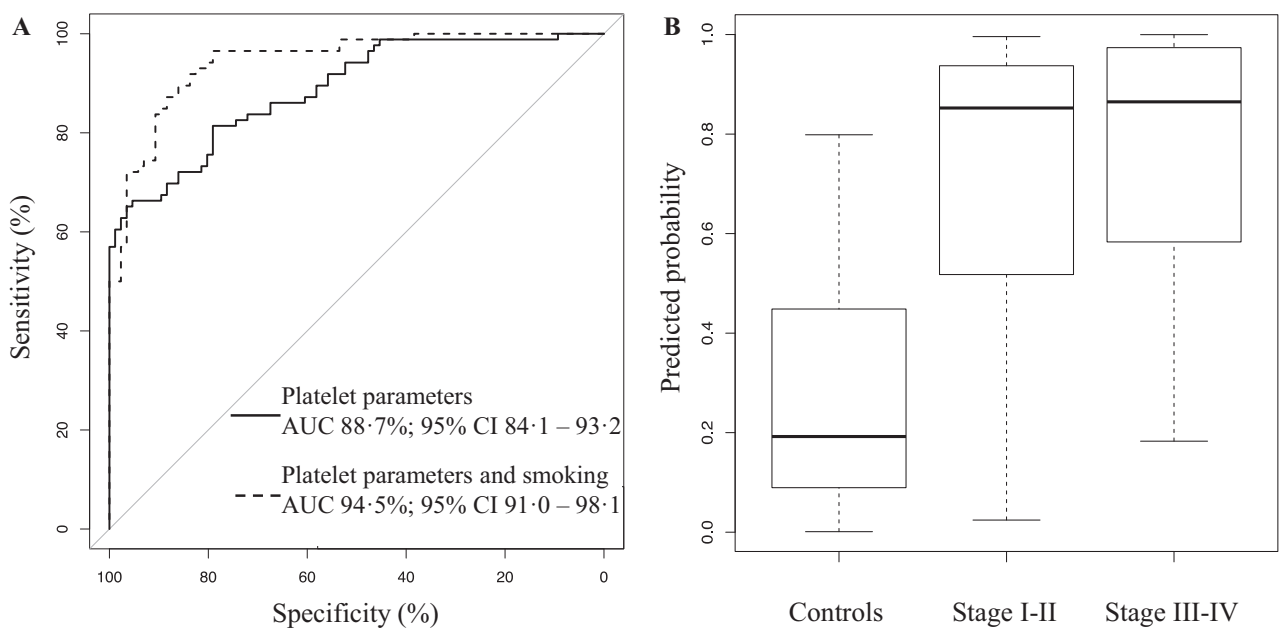

Fig. 2. A combination of platelet features allows discrimination between lung cancer patients and controls. The prediction model consists of a final set of six parameters (platelet count, MPV, PF4 and CTAPIII concentrations in platelets, and CTAPIII and TSP-1 concentrations in PFP). (A) Receiver operating characteristic (ROC) curves of the diagnostic model based on the platelet parameters (solid line) and after addition of smoking as a variable (hatched line). (B) Boxplot of the predicted probability using the diagnostic model based on the platelet parameters of lung cancer patients and controls. PF4, platelet factor 4; CTAPIII, connective tissue-activating peptide III; TSP-1, thrombospondin-1; MPV, mean platelet volume; PFP, platelet-free plasma. 

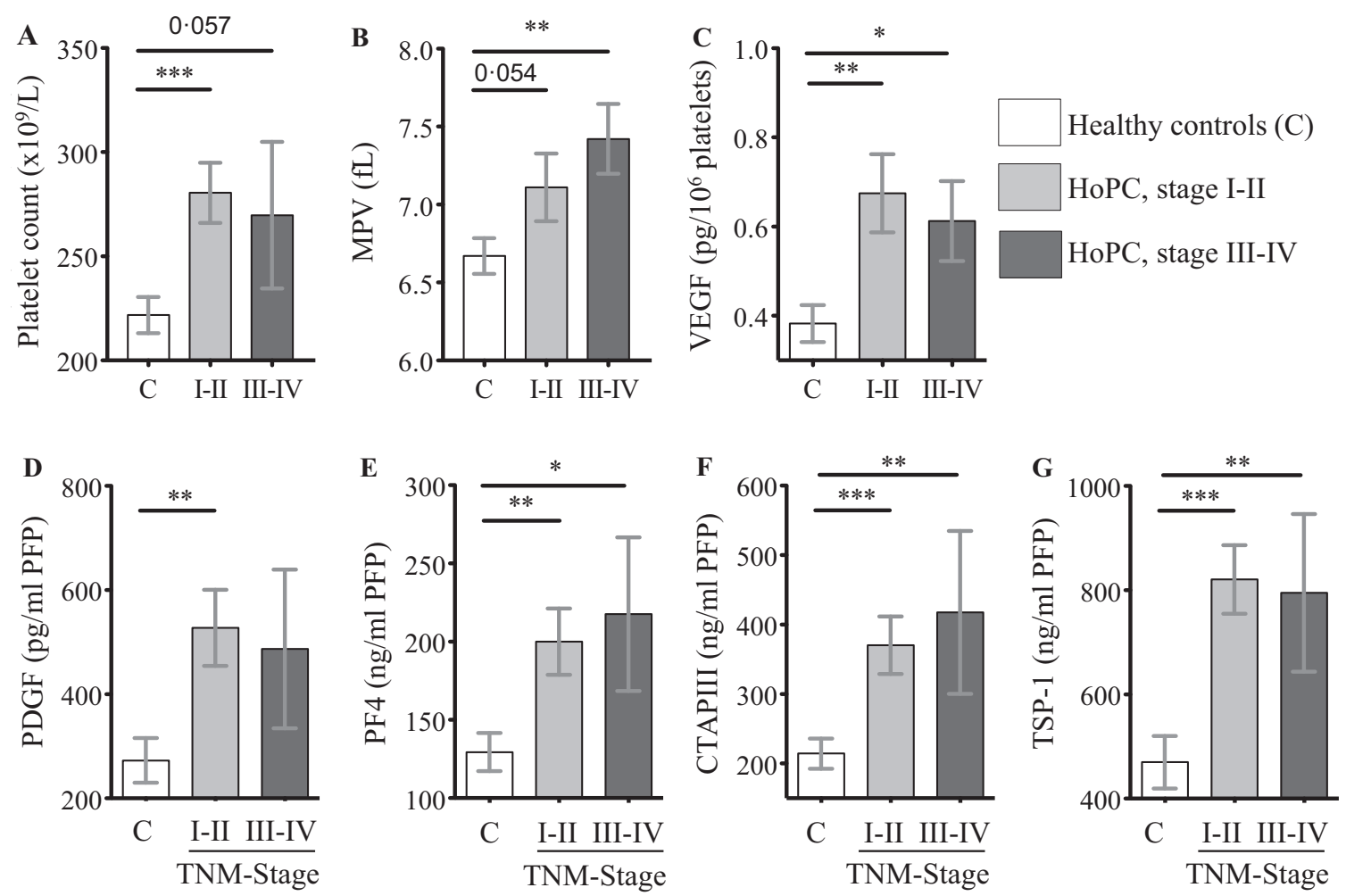

Fig. 3. Head of pancreas cancer (HoPC) is associated with changes in platelet count, mean platelet volume (MPV), increased platelet VEGF content and platelet activation. Platelet characteristics were measured in healthy controls $(\mathrm{C}, \mathrm{n}=42)$ and in patients with HoPC (subdivided according to TNM-stage: $\mathrm{I}-\mathrm{II}, \mathrm{n}=29$ and III-IV, $\mathrm{n}=13$ ). (A-B) Whole blood platelet count and MPV were measured after blood collection. Platelets and platelet-free plasma (PFP) were isolated from whole blood and concentrations of VEGF, PDGF, CTAPIII, PF4 and TSP-1 were determined. Only significantly changed parameters are shown: VEGF concentration in platelets (C), and PDGF, PF4, CTAPIII and TSP-1 concentrations in PFP (D-G). Data are presented as means with standard errors of the means. ${ }^{*} \mathrm{p}<0.05 ; * \mathrm{*}<0.01 ; * * \mathrm{p}<0.001$. TNM, tumour-node-metastasis; PDGF, platelet-derived growth factor; PF4, platelet factor 4; CTAPIII, connective tissue-activating peptide III; TSP-1, thrombospondin-1; VEGF, vascular endothelial growth factor.

concentration was significantly elevated in stage I-II only (Fig. 3D), while PF4, CTAPIII and TSP-1 concentrations were increased in plasma of patients with early- and latestage head of pancreas cancer (Fig. 3E-G). Strikingly, VEGF concentrations in PFP were not different between patients with head of pancreas cancer and healthy individuals (Supplemental Fig. 2E).

The stepwise backward elimination procedure yielded a diagnostic model for head of pancreas cancer consisting of three parameters (platelet count, MPV and VEGF concentration in platelets). Nagelkerke's $\mathrm{R}^{2}$ of this model was 0.418 , which is indicative of good model fit. The discriminative ability, as expressed by the AUC (Fig. 4A), was $82.7 \%$ (95\% CI 74.1-91.6). The optimism-corrected estimate of discriminative ability was $80.8 \%$. This parsimonious model discriminated well between patients with head of pancreas cancer and controls, as confirmed by the boxplots of predicted probability by the diagnostic model (Fig. 4B). Addition of smoking as a variable did not change the predicted probability of this diagnostic model (data not shown).

\section{Discussion}

The presence of a tumour in the human body appears to influence several platelet features. In this study, we identified changes in platelet count, volume and protein content in patients with lung or head of pancreas cancer as compared to healthy individuals matched for sex and age. In addition, circulating platelets appeared to be activated and plasma protein content was found to be different in cancer patients. Although a number of changes were similar in both patient groups, the precise change pattern appeared to be cancer type specific. Multivariable modelling of platelet characteristics enabled discrimination between lung or head of pancreas cancer patients, already at an early stage, and their healthy control groups.

Previous studies have already shown that tumours can affect platelet content of patients with cancer in different ways. This results from the fact that both platelets [12] and megakaryocytes [13] are able to actively sequester tumour-derived proteins from their microenvironment by endocytosis. We show an increase of VEGF in platelets of patients with lung and head of 

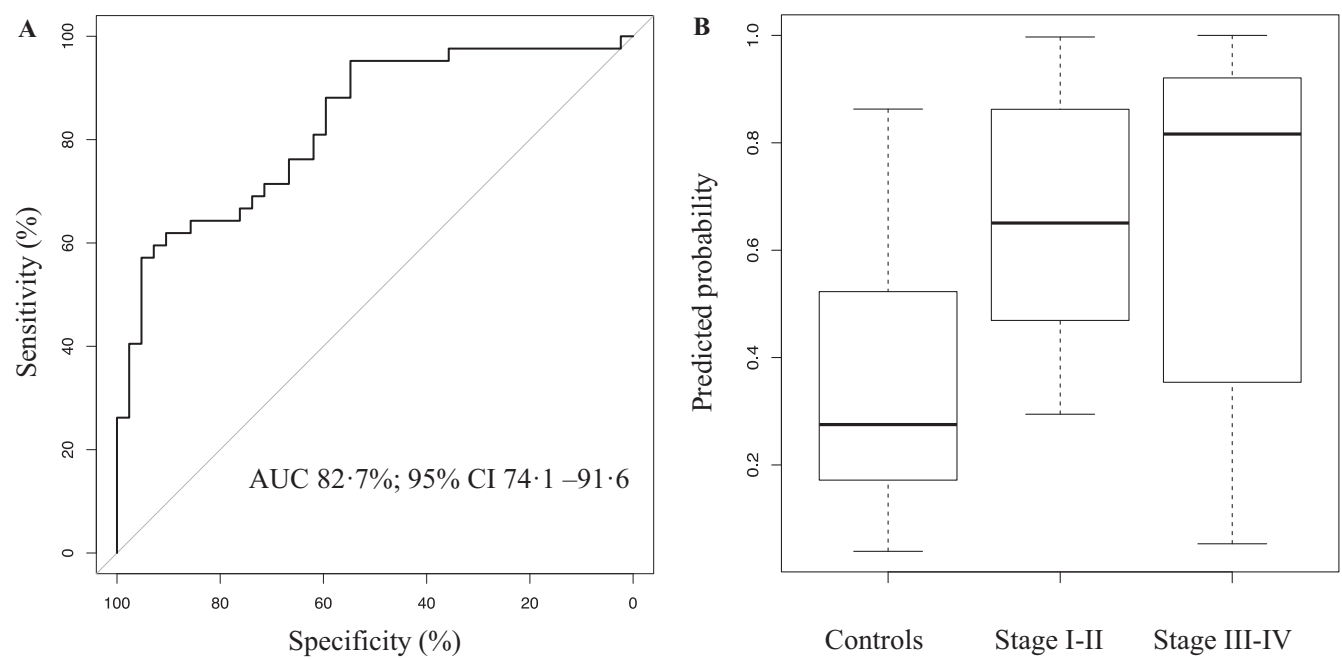

Fig. 4. A combination of platelet parameters allows discrimination between patients with head of pancreas cancer and controls. The prediction model consists of a final set of three parameters (platelet count, MPV and VEGF concentration in platelets). (A) Receiver operating characteristic (ROC) curve of the diagnostic model. (B) Boxplot of the predicted probability using the diagnostic model based on a combination of platelet parameters of patients with head of pancreas cancer and controls. VEGF, vascular endothelial growth factor; MPV, mean platelet volume.

pancreas cancer. This is in agreement with other studies demonstrating an increase of VEGF in platelets of patients with colon [4], liver [14,15] and lung [5] cancer. While platelet VEGF content appears to increase in all investigated cancer types, changes in other platelet growth factors in chemokines seem to be more cancer type dependent. For example, we found an elevated PDGF concentration in platelets of patients with earlystage lung cancer, as did Peterson et al. [4] in colorectal cancer patients. This contrasts with our findings in head of pancreas cancer patients and data from another study with hepatocellular carcinoma patients, indicating no changes in platelet PDGF concentration [14]. Furthermore, we found that PF4, CTAPIII and TSP-1 concentrations were reduced in platelets of patients with stage III-IV lung cancer, but not in platelets of patients with head of pancreas cancer. Hepatocellular carcinoma was reported to have no effect on platelet PF4 concentration [14], and non-metastatic colorectal cancer appeared to be accompanied by an increase in platelet PF4 [4]. Overall, the above data support the notion of tumour type dependency of platelet changes, an effect that - directly or indirectly via megakaryocytes - may be due to differences in cancer cell secretome [16].

The elevated PF4 and CTAPIII concentrations in plasma reveal the presence of activated platelets in the circulation of patients with lung or head of pancreas cancer. Also other studies used these parameters to demonstrate in vivo platelet activation in patients with cancer $[17,18]$. We excluded the possibility that the increased plasma concentrations were the result of ex vivo platelet activation during collection and handling of blood by measuring $\mathrm{P}$-selectin expression and integrin $\alpha_{\mathrm{IIb}} \beta_{3}$ activation on the surface of the platelets by whole blood flow cytometry. The fact that no differences between patients and controls were found and that platelets were still equally responsive to in vitro activation, confirms the notion that the elevated PF4 and CTAPIII concentrations in plasma are caused by platelet activation in the circulation of cancer patients. This can occur within the tumour vasculature, as well as systemically [19]. The leaky, irregular tumour vasculature creates a prothrombotic microenvironment by expressing proteins such as collagen and/or tissue factor, which are potent platelet activators [3]. In addition, cancer cells can secrete or express platelet-activating factors such as thrombin, adenosine diphosphate (ADP) and thromboxane $\mathrm{A}_{2}$ [20].

Similar to studies by others [7], we detected an increased platelet count in patients with cancer. Malignant cells produce thrombopoietic cytokines, leading to paraneoplastic thrombocytosis [8]. Our finding that MPV is elevated in peripheral blood of patients with cancer is also in agreement with earlier findings [21]. There is evidence indicating that larger platelets are metabolically and enzymatically more active than smaller platelets, resulting in higher in vivo platelet activation $[22,23]$. However, the exact underlying pathophysiological mechanism of the presence of larger platelets in cancer patients is not fully understood.

Altogether, our study shows that multiple platelet characteristics are changed in patients with cancer, both in early and later stages of development. Therefore, a multivariable diagnostic model was developed for both cancer types. Performance of the model in the lung cancer group, with six parameters (platelet count, MPV, PF4 and CTAPIII concentrations in platelets, and CTAPIII and TSP-1 concentrations in PFP), reached a high AUC of $88.7 \%$ (95\% CI 84.1-93.2). Addition of 
the independent risk factor smoking as a variable significantly increased the performance of the model, achieving an AUC of $94.5 \%$ (95\% CI 91.0-98.1). This also corrects for potential effects of smoking on platelet characteristics.

Exploiting the same strategy, another multivariable diagnostic model was created for patients with head of pancreas cancer, which included only three parameters (platelet count, MPV and VEGF concentration in platelets), but also performed well (AUC 82.7\%; 95\% CI 74.1-91.6). These findings strongly suggest that a combination of platelet indices can be used to distinguish patients with cancer from healthy individuals. Importantly, addition of a known risk factor such as smoking in case of lung cancer improves the discriminative power of such a model. The distribution of predicted probabilities of lung and head of pancreas cancer patients showed only limited overlap with that of the controls. This holds not only for stage III-IV patients but also for stage I-II patients, suggesting discriminating power of a combination of platelet features for early-stage cancer.

The diagnostic potential of platelets in cancer was recently also highlighted by a study of Best et al. [6], who demonstrated changes in platelet RNA profiles in patients with cancer, enabling discrimination between cancer patients and healthy individuals with high sensitivity and specificity. One important issue in this interesting study is the nature of the control group, that is clearly younger and with a different gender distribution than most of the cancer groups. Our data demonstrate statistically significant effects of both sex and age on various platelet characteristics, a finding that has been reported before in other studies as well $[24,25]$. This finding shows that inclusion of controls with similar sex and age is a key feature in studies like this. Another difference with our design is the fact that most patients in the study of Best et al. [6] are in an advanced stage of cancer, which leaves the question whether platelet RNA profiles offer diagnostic opportunities for early-stage cancer unanswered.

Overall, our study demonstrates that combinations of platelet features are potential biomarkers of cancer, even in patients with early-stage localised disease. The minimally invasive way to obtain platelets offers an attractive alternative to detect and diagnose cancer, as opposed to radiology and pathological analysis of tissue biopsies with all associated limitations. Future research is needed to further investigate the clinical relevance of our findings. Larger patient groups and additional cancer types have to be investigated. In addition, the effect of cancer treatment on platelet characteristics should be studied, to determine whether platelets could be employed to monitor the response to treatment. Platelets are a new and uncharted source of information, which need to be further explored in blood-based biomarker research.

\section{Funding}

The Netherlands Organisation for Scientific Research (project number 017.008.143, to SS).

\section{Conflict of interest statement}

None declared.

\section{Appendix A. Supplementary data}

Supplementary data related to this article can be found at http://dx.doi.org/10.1016/j.ejca.2017.04.010.

\section{References}

[1] DeSantis CE, Lin CC, Mariotto AB, Siegel RL, Stein KD, Kramer JL, et al. Cancer treatment and survivorship statistics, 2014. CA Cancer J Clin 2014;64:252-71.

[2] Sabrkhany S, Kuijpers MJ, Verheul HM, Griffioen AW, oude Egbrink MG. Platelets: an unexploited data source in biomarker research. Lancet Haematol 2015;2:e512-3.

[3] Sabrkhany S, Griffioen AW, Oude Egbrink MG. The role of blood platelets in tumor angiogenesis. Biochim Biophys Acta 2011;1815:189-96.

[4] Peterson JE, Zurakowski D, Italiano Jr JE, Michel LV, Connors S, Oenick M, et al. VEGF, PF4 and PDGF are elevated in platelets of colorectal cancer patients. Angiogenesis 2012;15: 265-73.

[5] Yao L, Dong H, Luo Y, Du J, Hu W. Net platelet angiogenic activity (NPAA) correlates with progression and prognosis of non-small cell lung cancer. PLoS One 2014;9:e96206.

[6] Best MG, Sol N, Kooi I, Tannous J, Westerman BA, Rustenburg F, et al. RNA-seq of tumor-educated platelets enables blood-based pan-cancer, multiclass, and molecular pathway cancer diagnostics. Cancer Cell 2015;28:666-76.

[7] Lin RJ, Afshar-Kharghan V, Schafer AI. Paraneoplastic thrombocytosis: the secrets of tumor self-promotion. Blood 2014;124: $184-7$.

[8] Stone RL, Nick AM, McNeish IA, Balkwill F, Han HD, Bottsford-Miller J, et al. Paraneoplastic thrombocytosis in ovarian cancer. N Engl J Med 2012;366:610-8.

[9] Mirsadraee S, Oswal D, Alizadeh Y, Caulo A, van Beek Jr E. The 7th lung cancer TNM classification and staging system: review of the changes and implications. World J Radiol 2012;4: $128-34$.

[10] Sabrkhany S, Kuijpers MJ, Verheul HM, Oude Egbrink MG, Griffioen AW. Optimal human blood sampling for platelet research. Curr Angiogenes 2013;2:157-61.

[11] Stryerberg E. Clinical prediction models: a practical approach to development, validation, and updating. 1st ed. Springer; 2009.

[12] Klement GL, Yip TT, Cassiola F, Kikuchi L, Cervi D, Podust V, et al. Platelets actively sequester angiogenesis regulators. Blood 2009;113:2835-42.

[13] Heijnen HF, Debili N, Vainchencker W, Breton-Gorius J, Geuze HJ, Sixma JJ. Multivesicular bodies are an intermediate stage in the formation of platelet alpha-granules. Blood 1998;91: $2313-25$.

[14] Alkozai EM, Porte RJ, Adelmeijer J, Zanetto A, Simioni P, Senzolo M, et al. Levels of angiogenic proteins in plasma and platelets are not different between patients with hepatitis $\mathrm{B} / \mathrm{C}$ related cirrhosis and patients with cirrhosis and hepatocellular carcinoma. Platelets 2015;26:577-82. 
[15] Kim SJ, Choi IK, Park KH, Yoon SY, Oh SC, Seo JH, et al. Serum vascular endothelial growth factor per platelet count in hepatocellular carcinoma: correlations with clinical parameters and survival. Jpn J Clin Oncol 2004:34:184-90.

[16] Pavlou MP, Diamandis EP. The cancer cell secretome: a good source for discovering biomarkers? J Proteomics 2010;73:1896-906.

[17] Al-Mondhiry H. beta-Thromboglobulin and platelet-factor 4 in patients with cancer: correlation with the stage of disease and the effect of chemotherapy. Am J Hematol 1983;14:105-11.

[18] Kaplan KL, Owen J. Plasma levels of beta-thromboglobulin and platelet factor 4 as indices of platelet activation in vivo. Blood 1981;57:199-202.

[19] Wojtukiewicz MZ, Sierko E, Kisiel W. The role of hemostatic system inhibitors in malignancy. Semin Thromb Hemost 2007;33: $621-42$

[20] Egan K, Crowley D, Smyth P, O'Toole S, Spillane C, Martin C, et al. Platelet adhesion and degranulation induce pro-survival and pro-angiogenic signalling in ovarian cancer cells. PLoS One 2011; 6:e26125.
[21] Pyo JS, Sohn JH, Kang G. Diagnostic and prognostic roles of the mean platelet volume in malignant tumors: a systematic review and meta-analysis. Platelets 2016;27:1-7.

[22] Thompson CB, Jakubowski JA. The pathophysiology and clinical relevance of platelet heterogeneity. Blood 1988;72:1-8.

[23] Mangalpally KK, Siqueiros-Garcia A, Vaduganathan M, Dong JF, Kleiman NS, Guthikonda S. Platelet activation patterns in platelet size sub-populations: differential responses to aspirin in vitro. J Thromb Thrombolysis 2010;30:251-62.

[24] Biino G, Santimone I, Minelli C, Sorice R, Frongia B, Traglia M, et al. Age- and sex-related variations in platelet count in Italy: a proposal of reference ranges based on 40987 subjects' data. PLoS One 2013;8:e54289.

[25] Panova-Noeva M, Schulz A, Hermanns MI, Grossmann V, Pefani E, Spronk HM, et al. Sex-specific differences in genetic and nongenetic determinants of mean platelet volume: results from the Gutenberg Health Study. Blood 2016;127:251-9. 